\title{
A novel method for comparing radiation dose and image quality, between and within different X-ray units in a series of hospitals
}

\author{
Sadeq Al-Murshedi ${ }^{1}$, Peter Hogg ${ }^{1}$, Luis Lanca ${ }^{2,3}$ and Andrew England ${ }^{1}$ \\ ${ }^{1}$ School of Health Sciences, University of Salford, Salford M6 6PU, United Kingdom. \\ ${ }^{2}$ Lisbon School of Health Technology, 1990-096 Lisbon, Portugal and Karolinska Institute, SE- \\ 17177 Stockholm, Sweden. \\ ${ }^{3}$ Singapore Institute of Technology, Health and Social Sciences Cluster, Singapore.
}

\section{ABSTRACT}

Objectives: To develop a novel method for comparing radiation dose and image quality (IQ) to evaluate adult chest X-ray (CXR) imaging among several hospitals.

Methods: CDRAD 2.0 phantom was used to acquire images in eight hospitals (17 digital X-ray units) using local adult CXR protocols. IQ was represented by image quality figure inverse $\left(\mathrm{IQF}_{\text {inv }}\right)$, measured using CDRAD analyser software. Signal to noise ratio (SNR), contrast to noise ratio (CNR) and conspicuity index (CI) were calculated as additional measures of IQ. Incident air kerma (IAK) was calculated using a solid-state dosimeter for each acquisition. Figure of merit (FOM) was calculated to provide a single estimation of IQ and radiation dose.

Results: IQ, radiation dose and FOM varied considerably between hospitals and X-ray units. For $\mathrm{IQF}_{\text {inv }}$, the mean (range) between and within the hospitals were $1.42(0.83-2.18)$ and 1.87 (1.522.18), respectively. For IAK, the mean (range) between and within the hospitals were 93.56 (17.26 to 239.15$) \mu \mathrm{Gy}$ and 180.85 (122.58-239.15) $\mu \mathrm{Gy}$, respectively. For FOM, the mean (range) between and within hospitals were 0.05 (0.01 to 0.14$)$ and 0.03 (0.02-0.05), respectively.

Conclusions: The suggested method for comparing IQ and dose using FOM concept along with the new proposed FOM, is a valid, reliable and effective approach for monitoring and comparing IQ and dose between and within hospitals. It is also can be beneficial for the optimisation of Xray units in clinical practice. Further optimisation for the hospitals /X-ray units with low FOM are required to minimise radiation dose without degrading IQ.

\section{Keywords}

Image quality, radiation dose, dose optimisation, adult chest radiography, CDRAD 2.0 phantom. 


\section{Introduction}

Dose Reference Levels (DRLs) are defined in legislation as a tool for monitoring radiation dose for X-ray imaging procedures [1,2]. However, DRLs are limited because they only take into account radiation dose and not image quality (IQ). Practically, DRLs can be beneficial for narrowing the likely variation in radiation dose within and between hospitals, however they cannot control the likely variation in IQ. The reason behind this is that reports in the literature have demonstrated no straightforward correlation between IQ and radiation dose between hospitals in different countries [3-6]. On the other hand, several studies [3-5] have attempted to assess the variation in IQ and patient dose between hospitals. These studies presented variations in IQ and radiation dose separately and did not combine them as a single metric. This makes comparisons of X-ray units difficult and restricts the determination of optimal parameters. Within radiography, there is always a trade-off between the radiation dose and the IQ by which no single factor should be analysed on its own. Utilising a figure of merit (FOM) offers an attractive way of providing a measure of IQ per unit dose. FOM values are commonly offered as a single number that reflects the efficiency of an imaging system under set conditions [7]. Consequently, using chest X-ray $(\mathrm{CXR})$ as a vehicle, this paper proposes a new method for comparing IQ per unit dose, within and between hospitals, based on a FOM concept. This paper also provides new information about variations in adult CXR radiation dose, IQ and clinical protocols variations between different Xray units.

\section{Materials and Methods}

The experiment was conducted using 17 diagnostic X-ray units located in 8 UK National Health Service (NHS) hospitals (Table 1). All X-ray units had been quality assessed using tests outlined in the Institute of Physics and Engineering in Medicine (IPEM) report 91[8]. Results indicated that the units were fit for routine clinical use; consequently, all the units were included in the study. 


\section{Contrast-detail phantom}

The CDRAD 2.0 phantom (Artinis Medical System, The Netherlands) was used to investigate contrast-detail performance (figure 1) [9]. The phantom consists of a square acrylic plastic plate $(265 \times 265 \mathrm{~mm})$, is $10 \mathrm{~mm}$ thick and has holes of various depths (contrasts) and diameters. It is divided into 255 squares, comprising 15 rows and 15 columns. The holes are logarithmically sized from 0.3 to $8.0 \mathrm{~mm}$ in both diameter and depth.

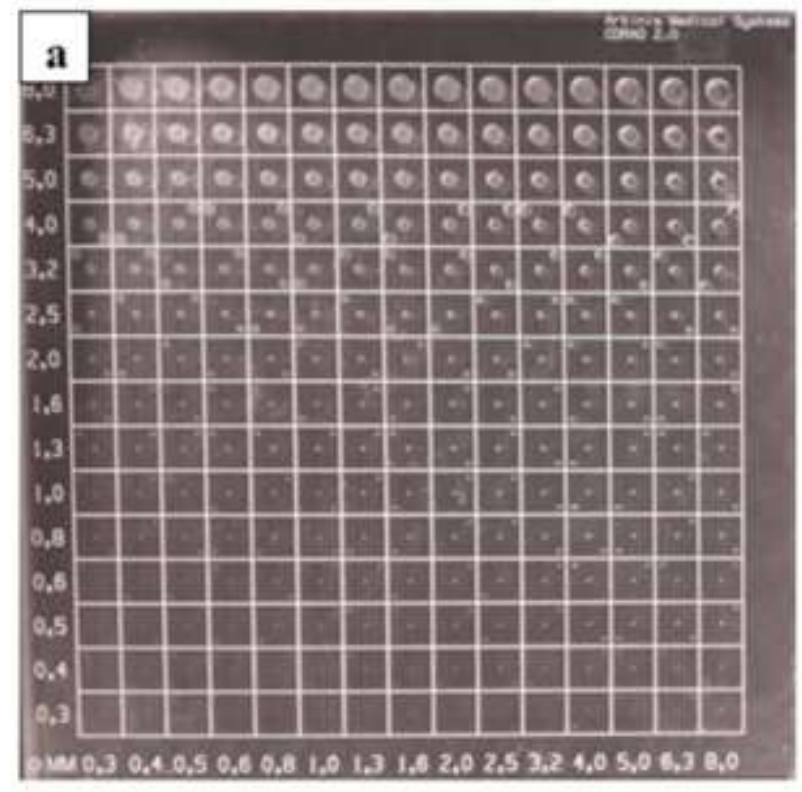

Figure 1a. CDRAD 2.0 phantom

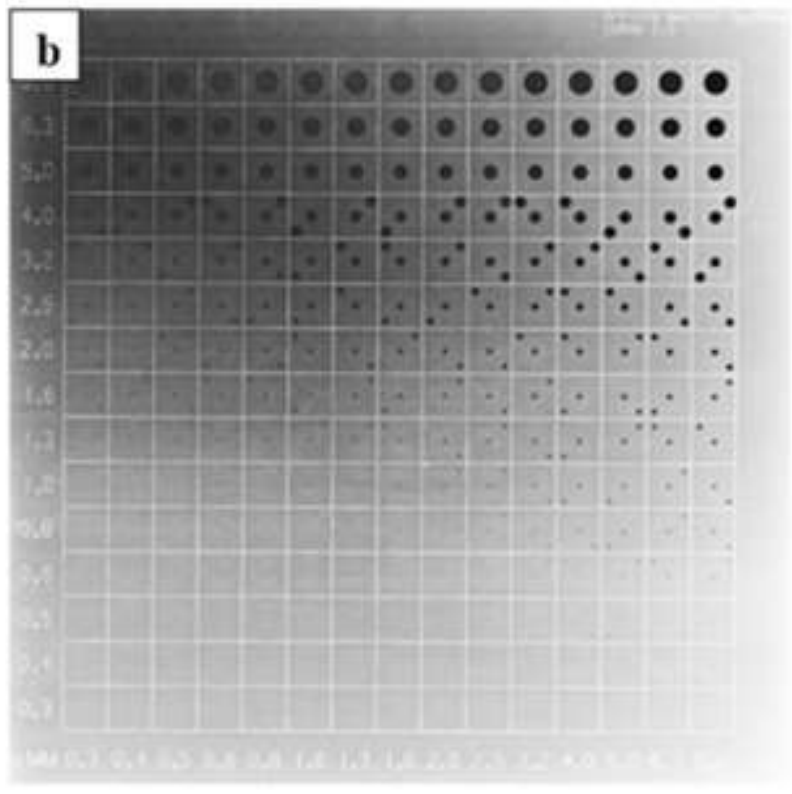

Figure 1b. Resultant CDRAD 2.0 X-ray image.

\section{Image acquisition}

The CDRAD 2.0 phantom was placed midway within $13 \mathrm{~cm}$ of polymethyl methacrylate (PMMA) slabs, to simulate the thickness of an adult chest. This attenuation property of PMMA was selected to be equivalent to an adult's trunk thickness $(20 \mathrm{~cm})$ [10] by applying a conversion factor at which each $1 \mathrm{~cm}$ of PMMA equates to a $1.5 \mathrm{~cm}$ thickness of the chest region [11,12]. The CDRAD 2.0 phantom was positioned at the centre of radiation field and the X-ray beam was collimated to the edges of the CDRAD 2.0 /PMMA phantom. Three images were acquired for each X-ray unit, as recommended by the CDRAD 2.0 phantom manufacturer [9]. CDRAD 2.0 /PMMA images were acquired on all 17 X-ray units using the local (default) adult CXR protocol as shown in Table 1. 


\section{Low contrast detail (LCD) detectability measurements}

The CDRAD analyser software was used for the physical evaluation of low contrast detail (LCD) detectability and the output of the software is displayed as image quality figure inverse (IQFinv), the average value of three repeated images. The $\mathrm{IQF}_{\text {inv }}$ value is based on equation (1):

$I Q F_{i n v}=\sum_{i=1}^{15} \frac{1}{C_{i} D_{(i, t h)}}$

Where: $\mathrm{D}_{(\mathrm{i}, \text { th) }}$ is the smallest visible hole diameter in column (i); $\mathrm{C}_{\mathrm{i}}$ is the depth of the hole in the column (i).

Prior to the analysis, the Alpha level of significance was set to be equal to $1 \mathrm{e}-8$, which is equal to the default value of the CDRAD analyser software and suggested by the manufacturer [9]. At this value the best correlation between the visual and physical IQFinv is observed [13]. The priori difference of means was set to ' 0 ' to ensure that there is a valid comparison between the images at different bit depth [9]. Finally, source image distance (SID) was set based on data collected from the local acquisition parameters.

\section{SNR, CNR and CI measurements}

SNR, CNR and CI were measured in the first square of the CDRAD 2.0 phantom image, which has the highest contrast and hole diameter $(8.00 \mathrm{~mm})$ [14]. The average pixel values of the central visible spot were considered as a signal, and the noise was measured from the standard deviation of the background. SNR was computed as the ratio between signal and noise ( $\left.\mathrm{SNR}=\mathrm{S} / \sigma_{\mathrm{b}}\right)$, this is considered a direct method for measuring SNR [15]. CNR was computed as the ratio of the average pixel values difference between signal and background divided by the noise value (CNR $\left.=\mathrm{S}-\mathrm{B} / \sigma_{\mathrm{b}}\right)[16,17]$. The computer software ImageJ (NIH, Bethesda, MD) was used to measure the SNR and CNR. CI was assessed by measuring the conspicuity change for the visible object (hole) from the first square of each image using the conspicuity software and equation (2) $[18,19]$ :

$C I=\frac{d \tan (\theta-1) \Delta G L}{\sqrt{\sigma_{s}^{2}+\sigma_{n}^{2}}}$ 
Where: $\mathrm{CI}=$ conspicuity index, $\mathrm{d}=$ maximum visible hole dimension, $\theta=$ maximum edge angle (the maximum slope angle to the edge of the visible hole profile) in degrees, $\Delta \mathrm{GL}=$ mean contrast (difference in grey level), $\sigma_{\mathrm{s}}=$ mean noise within the hole and $\sigma_{\mathrm{n}}=$ mean background noise.

\section{Radiation dosimetry}

A solid-state dosimeter (RaySafe X2, Unfors RaySafe AB, Billdal, Sweden) was used to measure IAK (without including backscatter radiation) [20] for the phantom and it was placed on the tubefacing side of the PMMA slabs in the centre of the X-ray field. We would like to confirm that the performance of the solid-state dosimeter was frequently checked by local medical physicists to ensure calibration to national standards. The solid-state dosimeter has a reported accuracy of $\pm 5 \%$ against the calibrated values. For the measurement of IAK, each exposure was repeated three times and the average value recorded. This method for measuring IAK was only used for radiographic examinations that were carried out using manual exposure control. For examinations which used the automatic exposure control (AEC) a simple mathematical technique was used to estimate IAK. This technique was based on the estimation the IAK values from the post-exposure $\mathrm{mAs}$ recorded at the point of acquisition. IAK values which corresponded to their respective mAs values were recorded using the solid-state dosimeter. From the graphical representation of IAK versus mAs, a best fit line and resultant regression equation were generated to provide a method for estimating the IAK from post-exposure mAs values obtained from the phantom.

\section{Statistical analysis}

SPSS software (version 22.0, IBM, Armonk, New York, USA) was used for analysing the data with the aim of identifying correlations. Data were first examined to determine normality using the Shapiro-Wilk test and also visually to determine the most appropriate correlation statistic. CI data were non-parametric, whilst IAK, IQF correlation was used (for parametric data) to investigate the correlation between IAK and the $\mathrm{IQF}_{\text {inv }}$, also used to investigate the correlation between $\mathrm{IQF}_{\text {inv }}$ and both CNR and SNR. Spearman's correlation was used to investigate the correlation between $\mathrm{IQF}_{\text {inv }}$ and CI. Investigating the degree of correlation between the IQFinv and IAK was necessary to assess the level of the optimisation across the X-ray machines. While, the correlations between the different physical IQ measures were useful to assess the relationship between them. A new formula of FOM was utilised, which 
for the first time, in conventional radiography, reports the $\mathrm{IQF}_{\text {inv }}$ per unit of radiation dose (IAK) and is calculated according equation 3 :

$$
\mathrm{FOM}=\left(\mathrm{IQF}_{\text {inv }}\right)^{2} / \mathrm{IAK}
$$

\section{Results}

Data on IQ are presented as a series of graphs. Hospitals and X-ray units are coded using two letters and one number: the letter $(\mathrm{H})$ refers to the hospital and the letter $(\mathrm{X})$ refers to the X-ray unit i.e. Hospital 1, X-ray unit 1 would be H1X1.Figure 2 illustrates the variation in IAK, between and within the hospitals. Figure 3 and figure 4 compare IQF $F_{\text {inv }}$ values against the corresponding IAK values, between and within the hospitals. Figures 5 to 7 illustrate the variation in physical IQ parameters CNR, SNR and CI, between and within the hospitals, respectively. Error bars represent the standard deviation (SD) obtained from taking three measurements. Finally, figure 8 illustrates the variation in FOM values among the X-ray units. These graphs show that there is considerable variation in the radiation dose and IQ values, both between and within the hospitals. A moderate (significant) correlation was observed between IAK and $\mathrm{IQF}_{\text {inv }}, \mathrm{r}=0.45(\mathrm{P}=0.02)$. Good (significant) correlations were observed between $\mathrm{IQF}_{\text {inv }}$ and both $\mathrm{CNR}$ and $\mathrm{CI}(\mathrm{r}=0.87(\mathrm{P}=0.001)$ and $\mathrm{r}=0.72(\mathrm{P}=0.001)$, respectively), while a weak non-significant correlation was observed with $\operatorname{SNR}(\mathrm{r}=0.05(\mathrm{P}=0.82))$. 


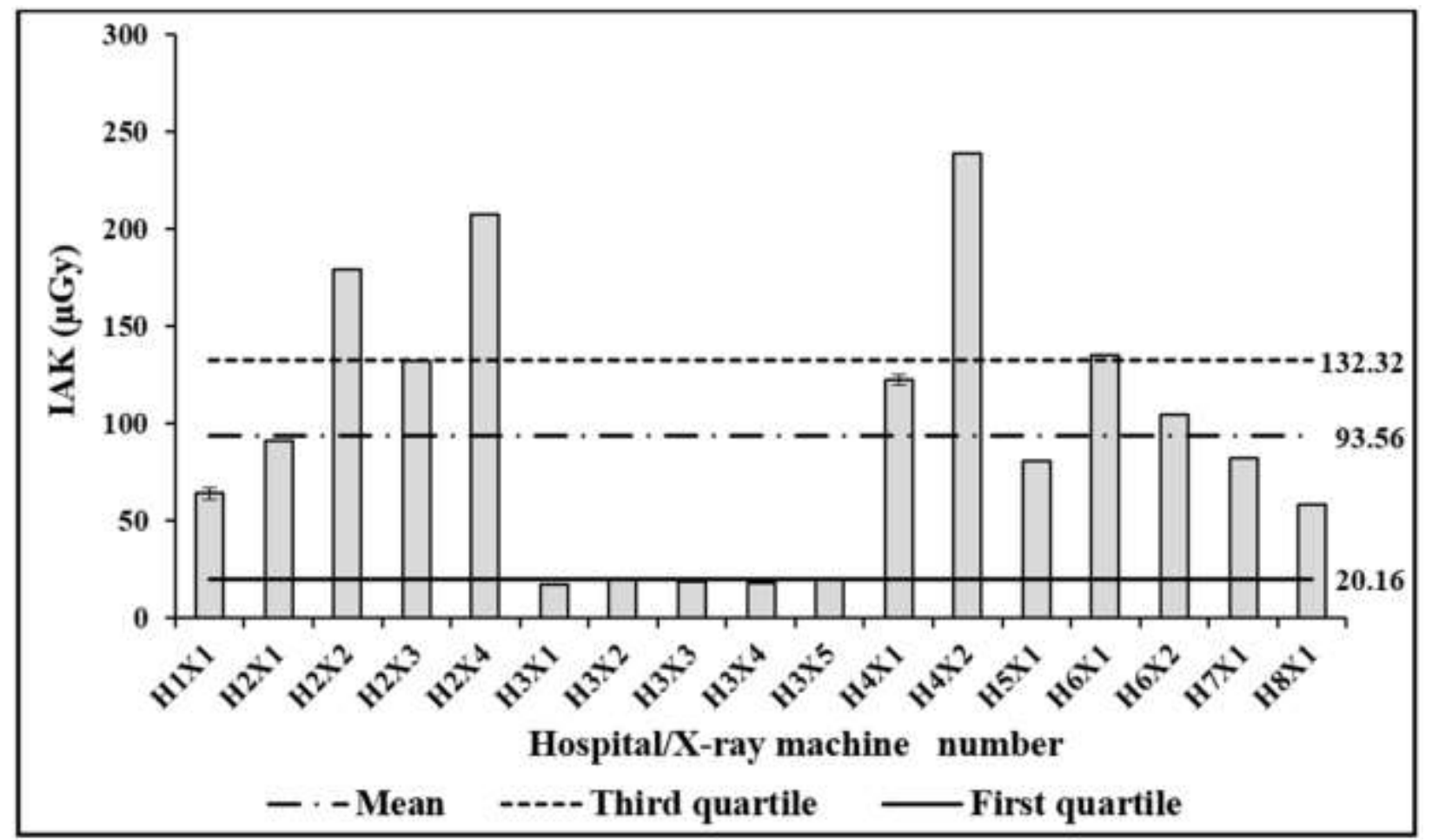

Figure 2. A bar chart displaying the variability of IAK values across the different X-ray units / hospitals.

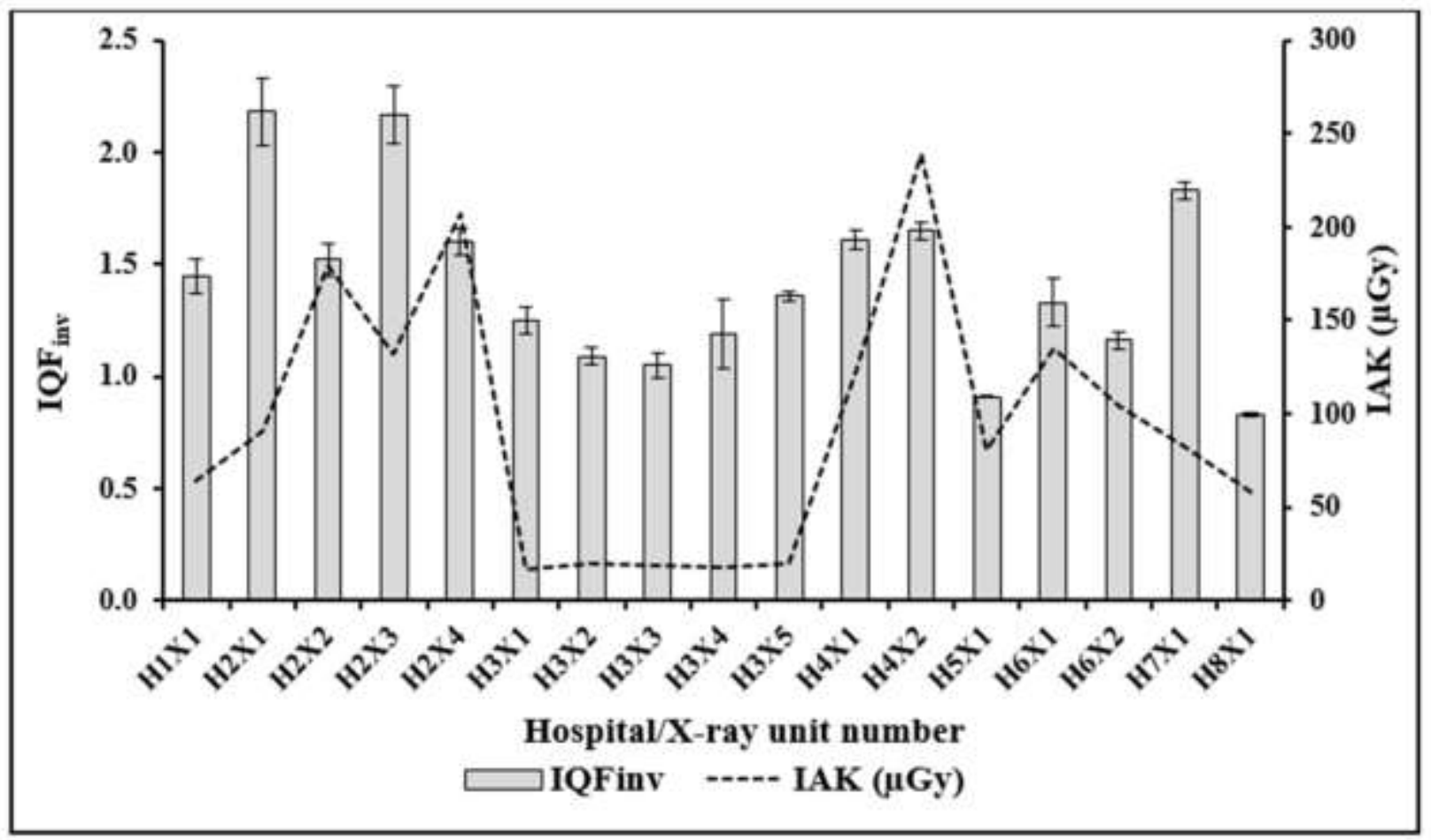

Figure 3. A comparison of IQFinv scores against the IAK values across the different X-ray units / 
hospitals.

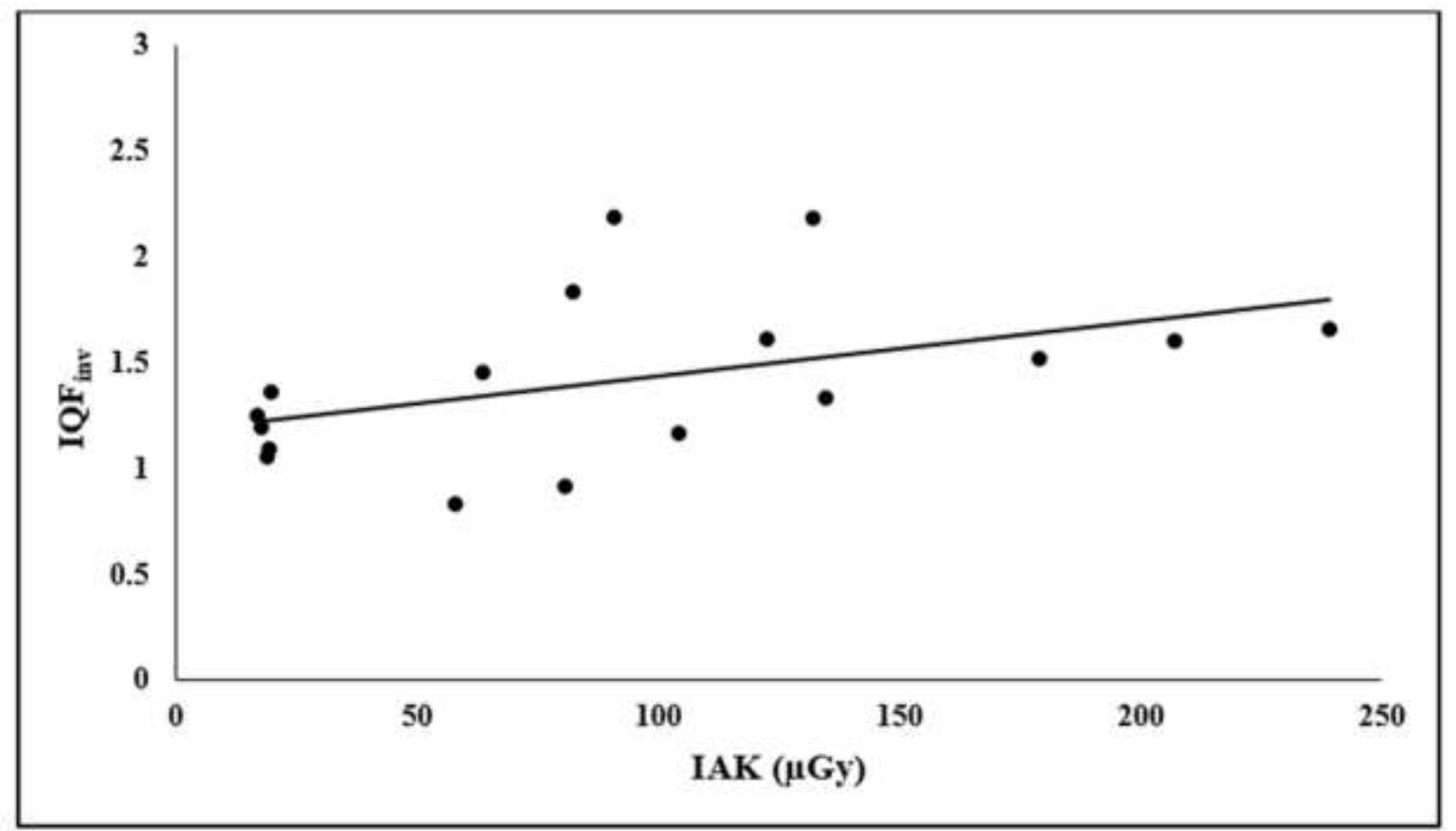

Figure 4. The scatterplot shows $\mathrm{IQF}_{\text {inv }}$ scores against the IAK values across the different $\mathrm{X}$-ray units / hospitals. 


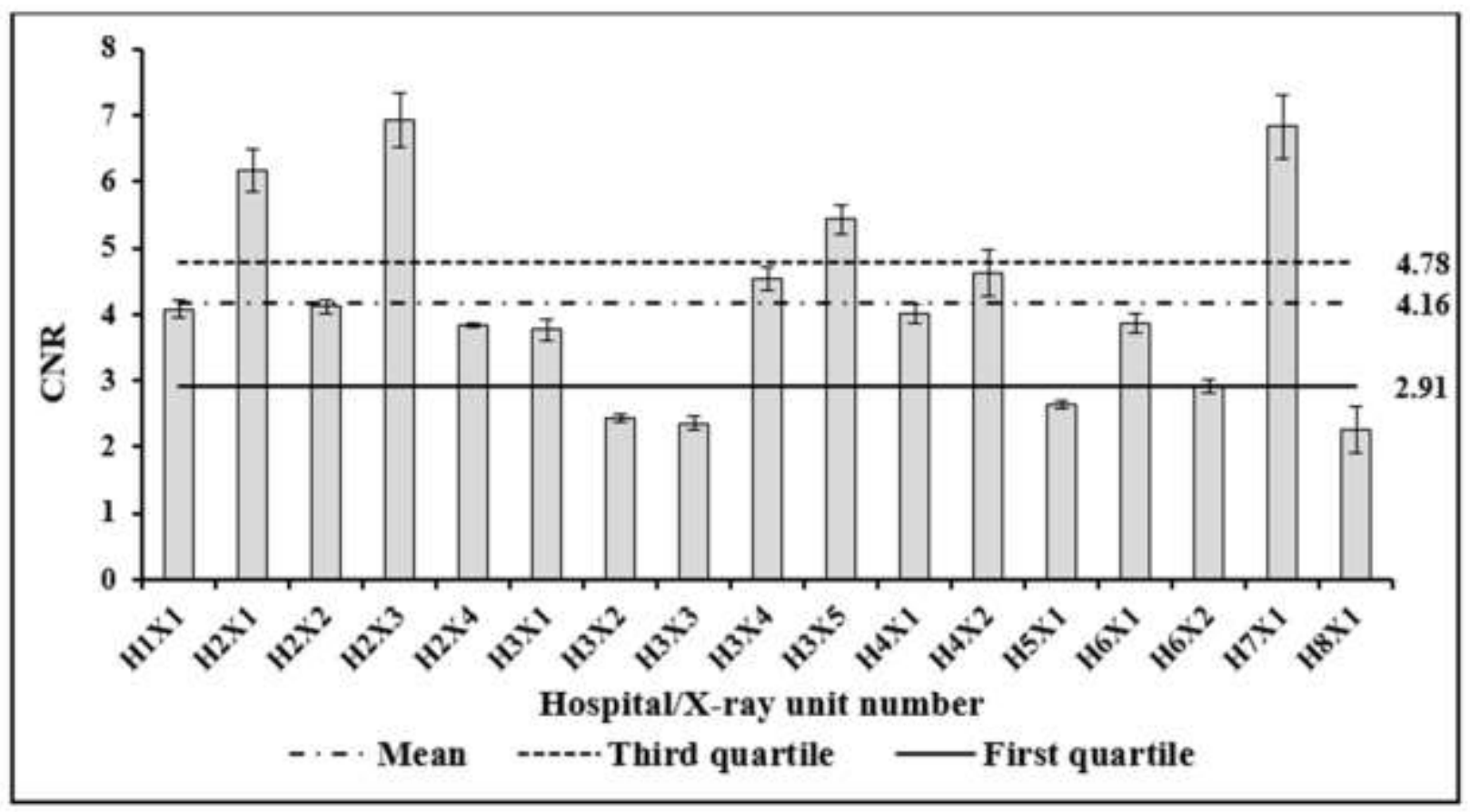

Figure 5. A bar chart displaying the variability of CNR values across the different X-ray units / hospitals.

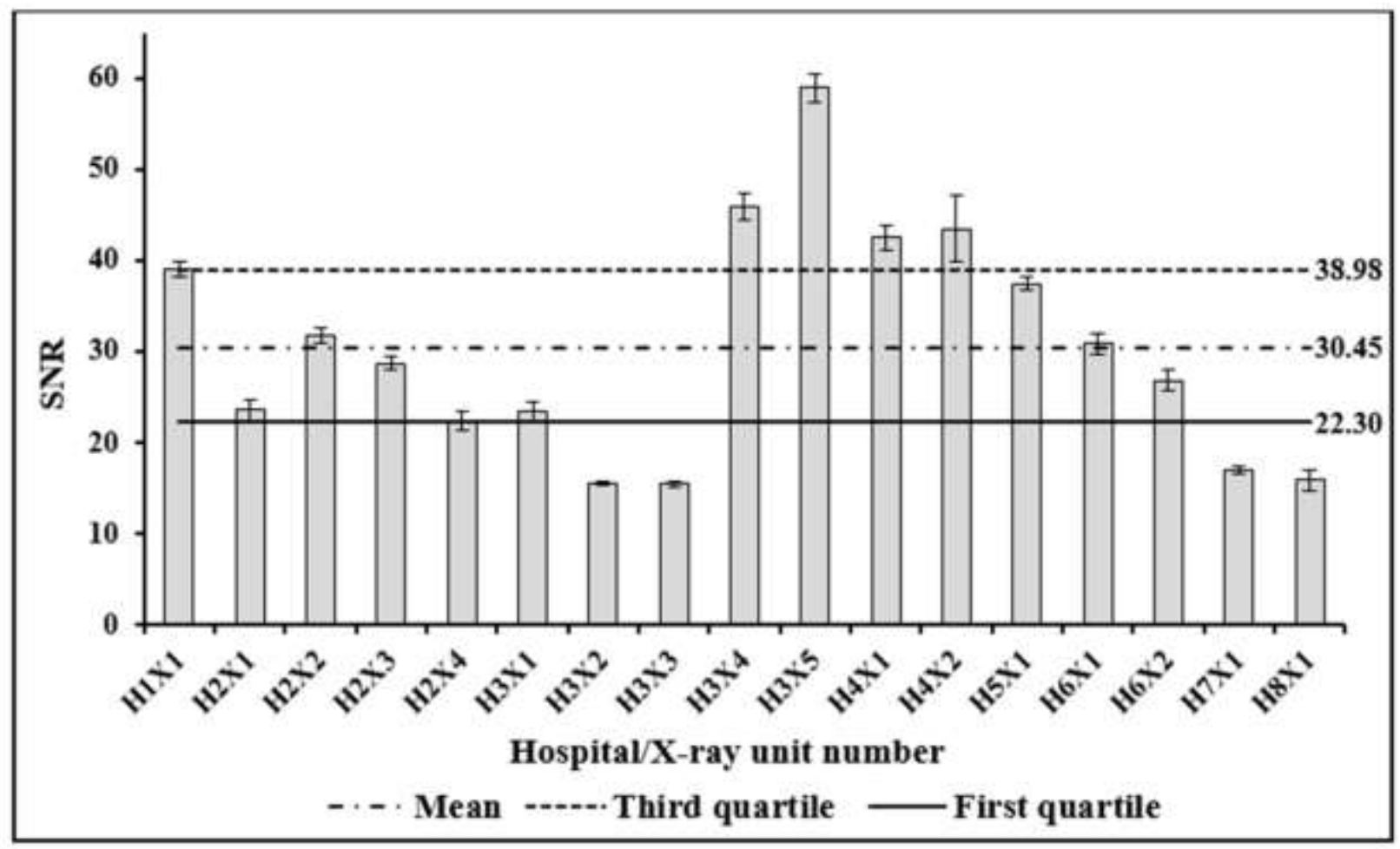

Figure 6. A bar chart displaying the variability of SNR values across the different X-ray units / hospitals. 


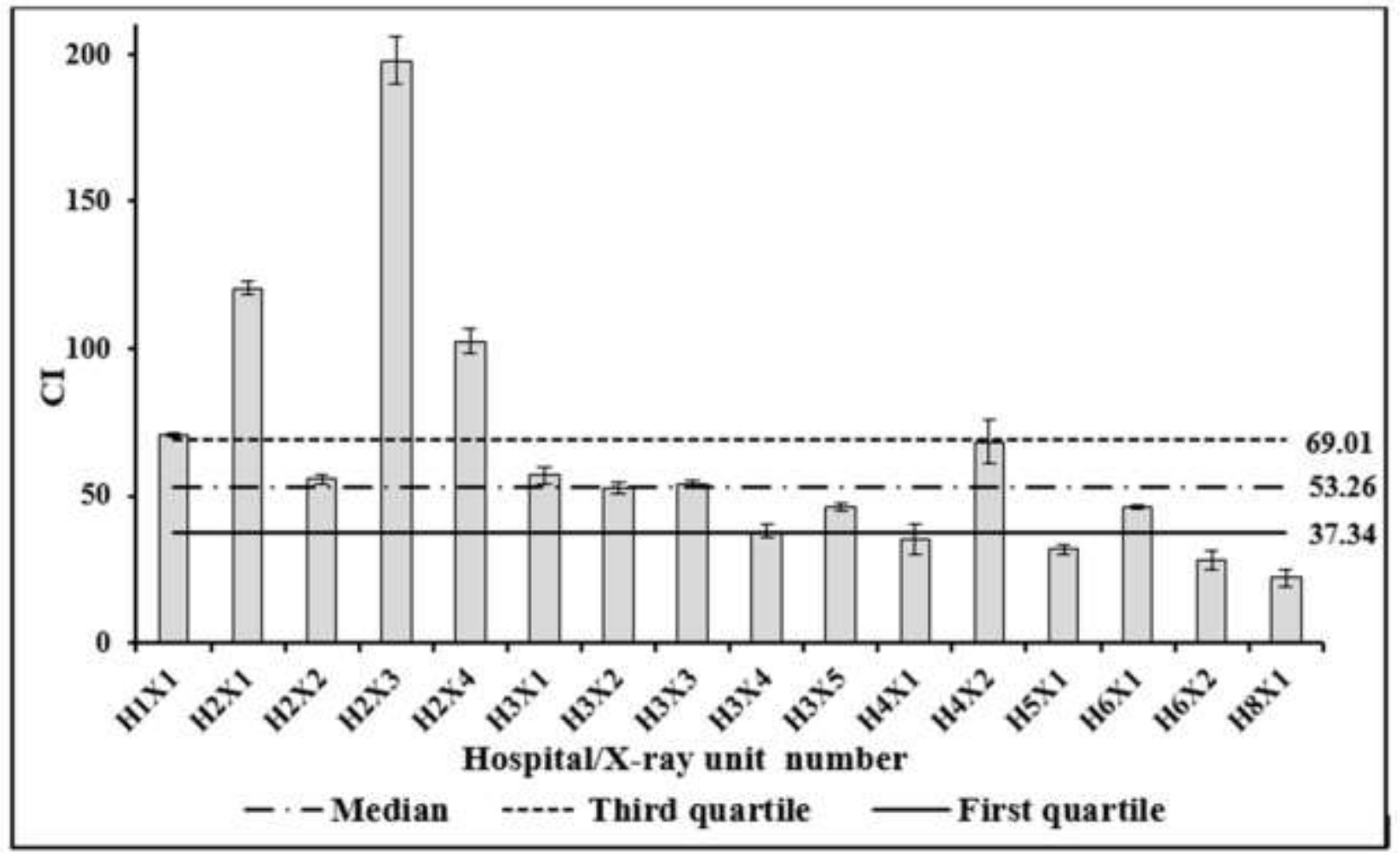

Figure 7. A bar chart displaying the variability of CI values across the different X-ray units / hospitals.

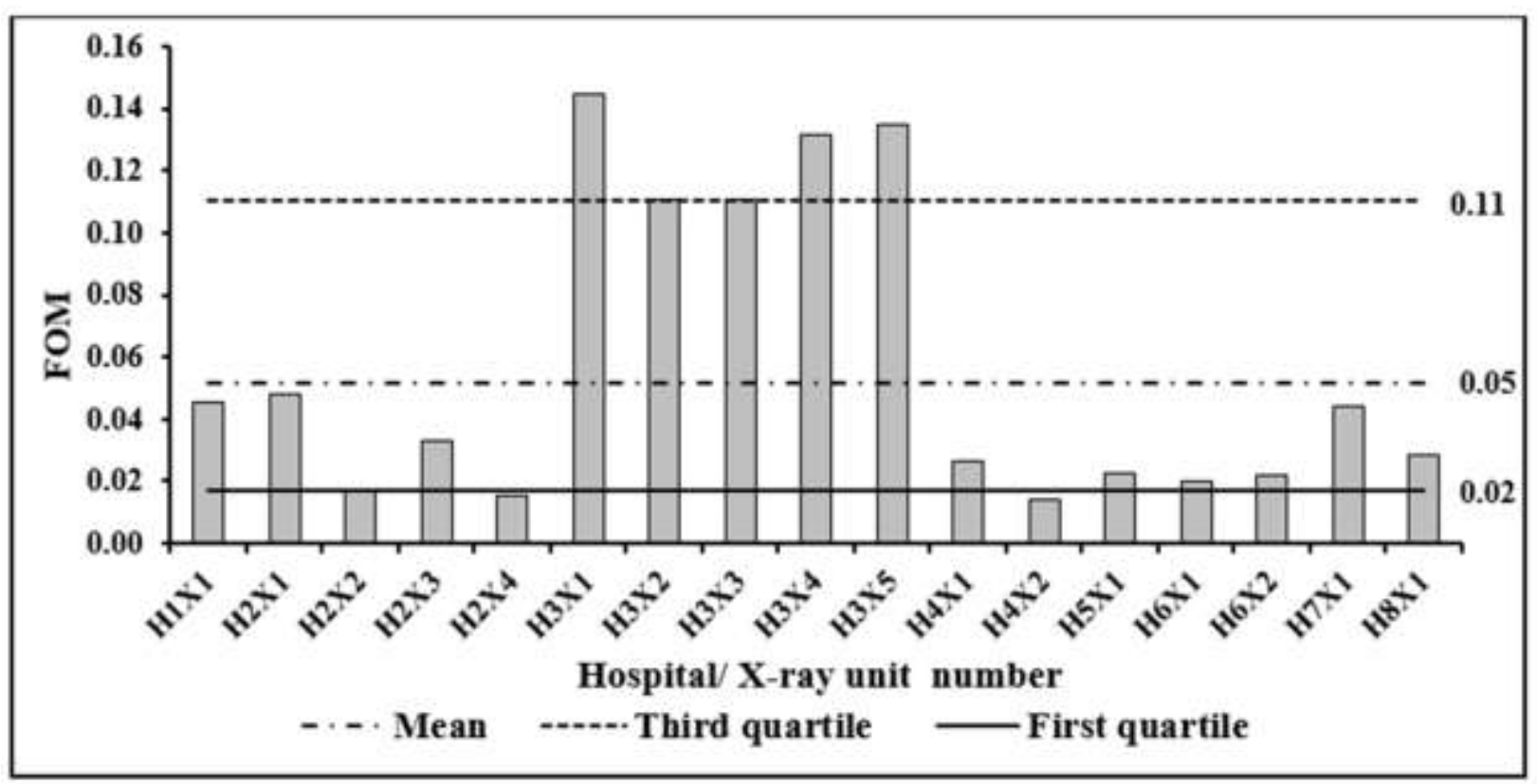

Figure 8. A bar chart displaying the variability of FOM values across the different X-ray units / hospitals. 
Table 1. Summary of X-ray equipment and their characteristics, examination techniques and acquisition parameters used for adult

CXRs between/within hospitals.

\begin{tabular}{|c|c|c|c|c|c|c|c|c|c|c|c|c|c|}
\hline $\begin{array}{l}\mathbf{H}^{1} \\
\text { No. }\end{array}$ & $\begin{array}{l}X^{2} \\
\text { No. }\end{array}$ & $\begin{array}{c}X \\
\text { type }\end{array}$ & $\begin{array}{l}D^{3} \\
\text { type }\end{array}$ & $\begin{array}{c}\mathbf{X} \\
\text { Manufacturer }\end{array}$ & $\begin{array}{c}\text { D } \\
\text { Manufacturer }\end{array}$ & $\begin{array}{l}\text { Type of } \\
\text { exposure } \\
\text { control }\end{array}$ & AEC & Grid & $\begin{array}{l}\text { Additional } \\
\text { filtration }\end{array}$ & $\begin{array}{l}\text { Focal } \\
\text { spot }\end{array}$ & $\begin{array}{l}\text { SID }^{8} \\
(\mathbf{c m})\end{array}$ & kVp & mAs \\
\hline 1 & 1 & Static & $\mathrm{IDR}^{4}$ & Philips & Philips & $\mathrm{AEC}^{7}$ & $\mathrm{R}+\mathrm{L}$ & yes & $\begin{array}{c}1.0 \mathrm{~mm} \\
\mathrm{Al}+0.1 \mathrm{~mm} \mathrm{Cu}\end{array}$ & Broad & 180 & 125 & 2.04 \\
\hline \multirow[t]{4}{*}{2} & 1 & Mobile & IDR & Carestream & Carestream & Manual & None & Yes & No & Broad & 180 & 110 & 2.80 \\
\hline & 2 & Static & IDR & Siemens & Siemens & AEC & $\mathrm{R}+\mathrm{L}$ & Yes & $0.1 \mathrm{~mm} \mathrm{Cu}$ & Broad & 180 & 125 & 2.10 \\
\hline & 3 & Static & $\mathrm{DDR}^{5}$ & Carestream & Carestream & AEC & $\mathrm{R}+\mathrm{L}$ & Yes & No & Broad & 180 & 125 & 2.88 \\
\hline & 4 & Static & IDR & Samsung & Samsung & $\mathrm{AEC}$ & $\mathrm{R}+\mathrm{L}$ & Yes & No & Broad & 180 & 125 & 4.40 \\
\hline \multirow[t]{5}{*}{3} & 1 & Static & IDR & Siemens & Siemens & Manual & None & No & $0.2 \mathrm{~mm} \mathrm{Cu}$ & Broad & 180 & 96 & 1.60 \\
\hline & 2 & Static & IDR & Siemens & Siemens & Manual & None & No & $0.2 \mathrm{~mm} \mathrm{Cu}$ & Broad & 180 & 96 & 1.60 \\
\hline & 3 & Static & IDR & Siemens & Siemens & Manual & None & No & $0.2 \mathrm{~mm} \mathrm{Cu}$ & Broad & 180 & 96 & 1.60 \\
\hline & 4 & Static & IDR & Siemens & Siemens & Manual & None & No & $0.2 \mathrm{~mm} \mathrm{Cu}$ & Broad & 180 & 96 & 1.60 \\
\hline & 5 & Static & IDR & Siemens & Siemens & Manual & None & No & $0.2 \mathrm{~mm} \mathrm{Cu}$ & Broad & 180 & 96 & 1.60 \\
\hline \multirow[t]{2}{*}{4} & 1 & Static & DDR & Philips & Philips & $\mathrm{AEC}$ & $\mathrm{R}+\mathrm{L}$ & Yes & $\begin{array}{c}1.0 \mathrm{~mm} \mathrm{Al} \\
+0.1 \mathrm{~mm} \mathrm{Cu}\end{array}$ & Fine & 180 & 125 & 3.13 \\
\hline & 2 & Static & $\mathrm{CR}^{6}$ & Philips & Carestream & AEC & $\mathrm{R}+\mathrm{L}$ & Yes & No & Fine & 180 & 125 & 5.50 \\
\hline 5 & 1 & Static & $\mathrm{CR}$ & Siemens & Carestream & Manual & None & No & No & Broad & 180 & 85 & 2.80 \\
\hline \multirow[t]{2}{*}{6} & 1 & Static & DDR & Philips & Philips & AEC & $\mathrm{R}+\mathrm{L}$ & Yes & No & Broad & 180 & 125 & 2.50 \\
\hline & 2 & Static & DDR & Philips & Philips & $\mathrm{AEC}$ & $\mathrm{R}+\mathrm{L}$ & Yes & No & Broad & 180 & 125 & 2.00 \\
\hline 7 & 1 & Static & DDR & Carestream & Carestream & $\mathrm{AEC}$ & $\mathrm{R}+\mathrm{L}$ & Yes & No & Broad & 180 & 125 & 1.80 \\
\hline 8 & 1 & Static & $\mathrm{CR}$ & Siemens & Carestream & Manual & None & No & No & Fine & 200 & 113 & 2.00 \\
\hline
\end{tabular}

$\mathrm{H}^{1}$ : Hospital; $\mathrm{X}^{2}$ : X-ray machine; $\mathrm{D}^{3}$ : Detector; $\mathrm{IDR}^{4}$ : indirect digital radiography; $\mathrm{DDR}^{5}$ : direct digital radiography; $\mathrm{CR}^{6}$ : computed radiography; $\mathrm{AEC}^{7}$ : automatic exposure control; $\mathrm{SID}^{8}$ : source to detector distance. 


\section{Discussion}

\section{Image quality}

LCD detectability is considered one of the most important issues in radiology, especially for CXRs since it gives an impression of the detection of certain types of lesions. The CDRAD 2.0 phantom has been widely utilised in the literature to evaluate LCD detectability for the purpose of optimisation and comparing the performance of imaging systems [4,14,21,22]. The physical evaluation of IQFinv was found to have a direct relationship with the visual IQ evaluations, with an excellent correlation published in a study by De Crop et al. [23]. Recently, a phantom study [24] concluded that the physical assessment of $\mathrm{IQF}_{\text {inv }}$ using CDRAD 2.0 phantom are a suitable option for visual IQ, visual lesion visibility evaluation and for CXR optimisation studies. Consequently, utilising CDRAD 2.0 phantom for IQ evaluation in our study is well justified.

Our study identified considerable variation in LCD detectability performance, between and within hospitals. The IQFinv scores between the hospitals ranged from 0.83 (H8X1) to 2.18 (H2X1) (mean 1.42). Within the hospitals the range was lower from 1.52 (H2X2) to 2.18 (H8X1) (mean 1.87); the third quartile between the hospitals was 1.61. It is clear from the distribution of IQFinv and IAK values (figures 2, 3 and 4) that there is a large variation in both metrics amongst the X-ray units. Only five out of seventeen X-ray units had $\mathrm{IQF}_{\text {inv }}$ scores higher than the $75^{\text {th }}$ percentile (1.61). Three X-ray units had $\mathrm{IQF}_{\text {inv }}$ scores lower than the $25^{\text {th }}$ percentile (1.16). The lower IQFinv observed in X-ray unit H8X1 had a value of 0.83 , this could be caused by the type of the image receptor (computed radiography $(\mathrm{CR}))$ and using a higher SID value $(200 \mathrm{~cm})$ when compared with the other X-ray units (Table 1). In addition, this X-ray unit (H8X1) did not make use of an anti-scatter grid and additional filtration when compared with some other X-ray units. Several LCD phantom studies have shown that the performance of digital radiography (DR) systems for IQ are significantly better than that of CR systems, for the same radiation dose level $[25,26]$. The reason behind the excellent performance of DR when compared with CR could be related to the high detective quantum efficiency (DQE) for DR systems compared with that of CR [27,28]. The reason behind the low DQE value for CR system is related to increased noise sources such as plate granularity and noise from the readout stage. The low DQE increases the visibility of noise on CR images compared with that of DR for the same employed exposure factors [29].

The type of detector $(\mathrm{CR})$ together with the relatively low $\mathrm{kVp}$ are likely to be the main reasons for the low $\mathrm{IQF}_{\text {inv }}$ score in X-ray unit H5X1 (lower than the $1^{\text {st }}$ quartile limit). From Table 1, it 
appears that the high $\mathrm{IQF}_{\text {inv }}$ score for X-ray unit $\mathrm{H} 2 \mathrm{X} 1$ is related to using manual exposure technique (with using anti-scatter grid) and using high mAs values when compared with other Xray units that used a manual exposure technique. A higher setting of mAs increases the number of the photons that reach the detector and this leads to an increase in SNR and a subsequent increase in the detection of the objects (visible holes of CDRAD 2.0 image). It has been demonstrated in previous contrast detail (CD) phantoms studies that there is a direct relationship between mAs and LCD detectability [30,31]. The use of AEC was observed to have an influence on the IQFinv scores. The mean (standard deviation (SD)) of $\mathrm{IQF}_{\text {inv }}$ scores, with and without AEC, were $1.59(\mathrm{SD}=0.29)$ and 1.23 ( $\mathrm{SD}=0.42$ ), respectively. This might be explained by considering all of the hospitals which used an AEC were associated with the use of the anti-scatter grid which improved the image contrast by reducing the scattered radiation reaching the detector. A CDRAD 2.0 phantom study was undertaken by Ween et al. who investigated anti-scatter grids and concluded that it does produce statistically significant differences on LCD detectability, when compared to not using a grid [32].

The other physical IQ measures (CNR, SNR and CI) also showed wide variation, both between and within hospitals. The CNR values between the hospitals ranged from 2.26 (H8X1) to 6.92 (H2X3) (mean 4.16). The within hospital range was $3.84(\mathrm{H} 2 \mathrm{X} 4)$ to $6.92(\mathrm{H} 2 \mathrm{X} 3)$ (mean 5.26). CI ranged from 22.12 (H8X1) to 197.88 (H2X3) (median 53.26) between hospitals. Within the hospitals, the range was smaller, 55.61(H2X2) to 197.88 (H2X3) (median 111.43). SNR ranged from $15.39(\mathrm{H} 3 \mathrm{X} 3)$ to $58.88(\mathrm{H} 3 \mathrm{X} 5)$ (mean 30.45) which was the same both between and within the hospitals. Similar to the IQFinv, the reason behind this variation in SNR, CNR and CI values is related to the variation of the image detectors types, X-ray machine characteristics and the differences in the acquisition parameters /protocols used among the X-ray units. Good (significant) correlations were observed between $\mathrm{IQF}_{\text {inv }}$ and both CNR and CI, while a weak nonsignificant correlation was observed with SNR. CNR values are frequently used as practical measures of object detection [33] and it is similar to the concept of signal to noise ratio that determines detection of the object in the Rose model [34]. Similarly, the CI represents the visibility of the hole (visible object), which also should have a good correlation with LCD detection. 


\section{Radiation exposure}

IAK was observed to vary both between and within hospitals (figure 2); IAK values between the hospitals ranged from 17.26 (H3X1) to $239.15 \mu \mathrm{Gy}$ (H4X2) (mean $93.56 \mu \mathrm{Gy}$ ). The IAK range for X-ray machines within the hospitals was smaller, 122.58 (H4X1) to 239.15 $\mu \mathrm{Gy}$ (H4X2) (mean $180.85 \mu \mathrm{Gy})$. The third quartile between the hospitals was $132.32 \mu \mathrm{Gy}$. The majority of the X-ray units (12) had IAK values lower than the $3^{\text {rd }}$ quartile $(132.32 \mu \mathrm{Gy})$. X-ray unit H4X2 was observed to have the highest IAK value $(239.15 \mu \mathrm{Gy})$. Table 1 shows the main reasons for the higher value in this X-ray unit could be attributed to the use of an AEC, with an anti-scatter grid and without any additional filtration. A CR system was also used in this X-ray unit and this has been shown to be an influencing factor on increasing the radiation exposure compared with DR systems. Several studies have shown that the performance of the DR system is significantly better than that of the CR system in terms of radiation dose reduction, with possible dose reduction of up to $75 \%$ in comparison with the CR system [25,35]. The lowest IAK values were observed in all five X-ray units in one hospital (H3) $(17.26,19.70,18.98,18.09$ and $20.16 \mu \mathrm{Gy})$ and this can be attributed to using a manual technique together with additional filtration $(0.2 \mathrm{~mm} \mathrm{Cu})$ and no anti-scatter grid. The results show that using the AEC has a high influence on IAK which increases considerably when using AEC. Mean IAK, with and without using AEC, were 140.72 (SD=57.68) and 40.50 $(\mathrm{SD}=31.25) \mu \mathrm{Gy}$, respectively. This high difference in the delivered IAK from the manual technique setting and that from AEC can be explained by considering that all the hospitals that used AEC were associated with the use of the anti-scatter grid. It was reported in the literature that using an anti-scatter grid increases the radiation dose significantly because a number of X-ray photons, part of the X-ray primary beam, are absorbed by the grid and this leads to an increase in the radiation dose to the patient by way of compensation [32].

\section{Image quality versus radiation dose/ FOM}

A FOM concept was utilised as a method for evaluating IQ and radiation dose in X-ray units, within and between hospitals, which we propose could be considered as a new method for monitoring purposes. Our FOM can be useful for optimisation of the X-ray units and imaging protocols. On the other hand, from the reviewed literature on optimisation, it was found that the common FOM formulas that are utilised with LCD detectability and a CD phantom such as CDRAD 2.0 phantom used are $\mathrm{FOM}=\mathrm{SNR}^{2} /$ dose and $\mathrm{FOM}=\mathrm{CNR}^{2} / \mathrm{dose}$ [36-38]. However, these two formulas use SNR and CNR as a metric which are self-limiting since, as stated, they do not 
take into account the object size (pathology or anatomical structure) during IQ evaluation [39] which is considered a limitation in these two measures of IQ. Several studies [40,41] have confirmed that the size of the lesions is important in their visualisation and detection in CXR. Therefore, a new formula of FOM that address this limitation is proposed in our work. In comparison with the existing FOM that have been utilised in the previous studies, the new FOM (equation 3) varies in two main aspects.1) it utilises the contrast detail as metric instead of SNR or CNR. This is particularly favours the detectability of lesions such as lung cancers in CXR. 2) another feature of the new FOM formula is that it takes into account the size of the objects (lesions or anatomical features) during IQ evaluations. The above two features are considered to be an advantage of the new FOM compared with those previously published in the literature. In addition, the LCD detectability represented by $\mathrm{IQF}_{\text {inv }}$ has been validated in a study undertaken by our research group [24] and was found to have a correlation with the visual measures of IQ and lesion visibility in CXR. Therefore, the suggested FOM in this paper (which utilise the IQFinv as a metric for IQ evaluation) is valid for IQ evaluation and optimisation studies. Another important feature of our new FOM is that it provides a standardised and reliable method for future comparisons of FOM values. Justification for this reason comes from the $\mathrm{IQF}_{\text {inv }}$ values generated from the CDRAD 2.0 phantom are exactly the same when the automated phantom analyser software is used (greater reliability). In contrast, other existing FOM formulas that utilise CNR or SNR as a metric for IQ evaluation and these could be difficult to compare between sites and machines. This is because there are many different techniques for measuring these physical measures, for example differences in the selection of the positions and sizes of the regions of interest (ROIs) for the measured objects and the background (noise). Based on this, the FOM can be influenced by the different techniques used for measuring the SNR or CNR, and then it might be difficult to compare the FOM values between X-ray units. Our FOM is based on using IQFinv, this can be compared between the different studies when using the same PMMA thicknesses.

The new FOM used in our study can also be supported by several recent optimisation studies in digital mammography that suggested utilising the $\mathrm{IQF}_{\text {inv }}$ from the CDMAM phantom as a measure for IQ. Within this work $\mathrm{IQF}_{\text {inv }}$ should be used in FOM calculations instead of the frequently used SNR and CNR $[42,43]$. To the best of our knowledge, our proposed and utilised method for evaluation and comparing the imaging systems performance, within and between hospitals, based on a FOM concept that has not been used before. 
FOM (figure 8) was observed to vary both between and within hospitals; the FOM values between hospitals ranged from 0.01 (H4X2) to 0.14 ( $\mathrm{H} 3 \mathrm{X} 1$ ) (mean 0.05), while the FOM within the hospitals ranged from $0.02(\mathrm{H} 2 \mathrm{X} 4)-0.05(\mathrm{H} 2 \mathrm{X} 1)$ (mean 0.03). The third quartile between the hospitals was 0.11 . Most of the FOM values of X-ray units (11) were between the mean and the $1^{\text {st }}$ quartile value (0.02). The high values of FOM for the five X-ray units in hospital 3 $(0.14,0.11,0.11,0.14$ and 0.14$)$ has a large influence on the level of the $3^{\text {rd }}$ quartile, mean and $1^{\text {st }}$ quartile and this lead to the values of FOM for majority of the X-ray units (12) failing to meet the mean (0.05). It was noticed that there are many variables in the existing protocols when acquiring CXR images and this could make it hard to determine the true factors causing these performance differences. However, it is clear from figure 8 that using DR with a manual exposure control, without an anti-scatter radiation grid and with additional filtration appears to be the optimal technique for reducing dose and maximising LCD detectability. It is suggested that other X-ray units, which have low values of FOM, especially lower than the $1^{\text {st }}$ quartile (i.e. H4X2) should consider reviewing their protocols for CXR examinations as this could lead to radiation dose reductions and protocol optimisation.

The inclusion of FOM, in addition to IQ and dose data provides valuable additional insight into variations in dose between X-ray units and hospitals, which would otherwise go undetected. The likely cause of the high variations in values of FOM between and within X-ray units can be attributed to: 1) differences in acquisition parameters and techniques/protocols; 2) technical characteristics of the detector technology in use. A moderate correlation $(r=0.45)$ between IAK and $\mathrm{IQF}_{\text {inv }}$ was observed and it was further identified that good IQ $\left(\mathrm{IQF}_{\text {inv }}\right)$ is not necessarily related to a high radiation dose. This may provide further evidence that there is opportunity for further optimisation of the radiographic technique and significant potential for radiation dose reduction without degrading IQ.

\section{Conclusion}

In this paper, a new method is proposed for comparing and optimising IQ and radiation dose in diagnostic radiography, between and within hospitals. Applying our method, based on the FOM concept could be beneficial for comparing and optimising the protocols used for the same radiography examination. Our new FOM, which utilises $\mathrm{IQF}_{\text {inv }}$ as a metric for IQ evaluation, is 
considered to be an effective, valid and reliable approach for comparing and optimising IQ and dose among X-ray units.

It is recommended that a FOM should be published for each hospital / X-ray machine and each radiographic examination. This will provide information on the level of variation in IQ and dose between X-ray units. Using this approach would be an improvement on current methods, which currently rely solely on DRLs.

\section{Acknowledgements}

The author is supported by the Higher Committee for Education Development in Iraq (HCEDIraq) and he gratefully acknowledges this supporting.

We have no conflicts of interest to declare. 


\section{References}

[1] Martin C J and Vano E 2018 Diagnostic reference levels and optimisation in radiology: Where do we go from here? J. Radiol. Prot. 38 E1-4

[2] International Atomic Energy Agency 2012 Radiation Protection in Paediatric radiology (Vienna)

[3] Geleijns J, Schultze Kool L J, Zoetelief J, Zweers D and Broerse J J 1993 Image Quality and Dosimetric Aspects of Chest X Ray Examinations: Measurements with Various Types of Phantoms Radiat. Prot. Dosimetry 49 83-8

[4] Veldkamp W J H, Kroft L J M, Boot M V., Mertens B J A and Geleijns J 2006 Contrastdetail evaluation and dose assessment of eight digital chest radiography systems in clinical practice Eur. Radiol. 16 333-41

[5] Almén A, Lööf M and Mattsson S 1996 Examination Technique, Image Quality, and Patient Dose in Paediatric Radiology Acta radiol. 37 337-42

[6] Struelens L, Hambach L, Buls N, Smans K, Malchair F, Hoornaert M T, Vanhavere F and Bosmans H 2008 Image quality assessment using the CD-DISC phantom for vascular radiology and vascular surgery Eur. J. Radiol. 67 348-56

[7] Borg M, Badr I and Royle G 2012 The use of a figure-of-merit (FOM) in digital mammography. Radiat. Prot. Dosimetry 151 81-8

[8] Scally A J 2005 Recommended Standards for the Routine Performance Testing of Diagnostic X-Ray Systems

[9] Burght R van der, Floor M, Thijssen M and Bijkerk R 2014 Manual CDRAD 2.0 phantom \& Analyser software version 2.1

[10] Tapiovaara M and, Siiskonen T 2008 PCXMC, A Monte Carlo program for calculating patient doses in medical $x$-ray examinations. (Helsinki)

[11] Vano E, Martinez D, Fernandez J M, Ordiales J M, Prieto C, Floriano a and Ten J I 2008 Paediatric entrance doses from exposure index in computed radiography. Phys. Med. Biol. $533365-80$

[12] Rassow J, Schmaltz A A, Hentrich F and Streffer C 2000 Effective doses to patients from paediatric cardiac catheterization. Br. J. Radiol. 73 172-83

[13] Pascoal a, Lawinski C P, Honey I and Blake P 2005 Evaluation of a software package for automated quality assessment of contrast detail images - comparison with subjective visual assessment Phys. Med. Biol. 50 5743-57

[14] Alzimami K, Sassi S, Alkhorayef M, Britten A J and Spyrou N M 2009 Optimisation of computed radiography systems for chest imaging Nucl. Instruments Methods Phys. Res. Sect. A Accel. Spectrometers, Detect. Assoc. Equip. 600 513-8

[15] Smans K, Struelens L, Smet M, Bosmans H and Vanhavere F $2010 \mathrm{Cu}$ filtration for dose reduction in neonatal chest imaging Radiat. Prot. Dosimetry 139 281-6 
[16] Hampel J R and Pascoal A 2018 Comparison and optimization of imaging techniques in suspected physical abuse paediatric radiography Br. J. Radiol. 20170650

[17] Jiang X, Baad M, Reiser I, Feinstein K A and Lu Z 2016 Effect of comfort pads and incubator design on neonatal radiography Pediatr. Radiol. 46 112-8

[18] Manning D J, Ethell S C and Donovan T 2004 Detection or decision errors? Missed lung cancer from the posteroanterior chest radiograph Br. J. Radiol. 77 231-5

[19] Szczepura K R and Manning D J 2016 Validated novel software to measure the conspicuity index of lesions in DICOM images International Society for Optics and Photonics. ed C K Abbey and M A Kupinski p 978703

[20] Zoetelief J, , Dance D R, , Drexler G, , Järvinen H and and Rosenstein M 2005 PATIENT DOSIMETRY FOR X RAYS USED IN MEDICAL IMAGING J. Int. Comm. Radiat. Units Meas. 5

[21] Geijer H, Beckman K-W, Andersson T and Persliden J 2001 Image quality vs radiation dose for a flat-panel amorphous silicon detector: a phantom study Eur. Radiol. 11 1704-9

[22] Rong X J, Shaw C C, Liu X, Lemacks M R and Thompson S K 2001 Comparison of an amorphous silicon/cesium iodide flat-panel digital chest radiography system with screen/film and computed radiography systems - A contrast-detail phantom study Med. Phys. 28 2328-35

[23] De Crop A, Bacher K, Van Hoof T, Smeets P V, Smet B S, Vergauwen M, Kiendys U, Duyck P, Verstraete K, D'Herde K and Thierens H 2012 Correlation of Contrast-Detail Analysis and Clinical Image Quality Assessment in Chest Radiography with a Human Cadaver Study Radiology 262 298-304

[24] Al-Murshedi S, Hogg P and England A 2018 An investigation into the validity of utilising the CDRAD 2.0 phantom for optimisation studies in digital radiography Br. J. Radiol. 20180317

[25] Hamer O W, Völk M, Zorger Z, Feuerbach S and Strotzer M 2003 Amorphous silicon, flatpanel, x-ray detector versus storage phosphor-based computed radiography: contrast-detail phantom study at different tube voltages and detector entrance doses. Invest. Radiol. 38 212-20

[26] Bacher K, Smeets P, Bonnarens K, De Hauwere A, Verstraete K and Thierens H 2003 Dose Reduction in Patients Undergoing Chest Imaging: Digital Amorphous Silicon Flat-Panel Detector Radiography Versus Conventional Film-Screen Radiography and Phosphor-Based Computed Radiography Am. J. Roentgenol. 181 923-9

[27] Bertolini M, Nitrosi A, Rivetti S, Lanconelli N, Pattacini P, Ginocchi V and Iori M 2012 A comparison of digital radiography systems in terms of effective detective quantum efficiency Med. Phys. 392617

[28] Schaefer-Prokop C, Neitzel U, Venema H W, Uffmann M and Prokop M 2008 Digital chest radiography: an update on modern technology, dose containment and control of image quality Eur. Radiol. 18 1818-30 
[29] McEntee M, Frawley H and Brennan P C 2007 A comparison of low contrast performance for amorphous Silicon/caesium iodide direct radiography with a computed radiography: A contrast detail phantom study Radiography 13 89-94

[30] Norrman E, Gårdestig M, Persliden J and Geijer H 2005 A Clinical Evaluation of the Image Quality Computer Program, CoCIQ J. Digit. Imaging 18 138-44

[31] Precht H, Tingberg A, Waaler D and Outzen C B 2014 New Developed DR Detector Performs Radiographs of Hand, Pelvic and Premature Chest Anatomies at a Lower Radiation Dose and/or a Higher Image Quality J. Digit. Imaging 27 68-76

[32] Ween B, Olstad M, Jakobsen J A and Olsen D R 2009 Pediatric digital chest radiography, comparison of grid versus non-grid techniques Eur. J. Radiogr. $1201-6$

[33] Moore C S, Wood T J, Beavis A W and Saunderson J R 2013 Correlation of the clinical and physical image quality in chest radiography for average adults with a computed radiography imaging system Br. J. Radiol. 8620130077

[34] Rose A 1948 The sensitivity performance of the human eye on an absolute scale. J. Opt. Soc. Am. 38 196-208

[35] Jansson M, Geijer H, Persliden J and Andersson T 2006 Reducing dose in urography while maintaining image quality - a comparison of storage phosphor plates and a flat-panel detector Eur. Radiol. 16 221-6

[36] Bosmans H, Nens J, Delzenne L, Marshall N, Pauwels H, De Wever W and Oyen R 2012 Exploration of exposure conditions with a novel wireless detector for bedside digital radiography Proceedings of International Conference "Medical Physics in the Baltic States” vol 8313, ed N J Pelc, R M Nishikawa and B R Whiting p 83135K

[37] Dragusin O, Smans K, Jacobs J, Inal T and Bosmans H 2008 Evaluation of the contrastdetail response of a cardiovascular angiography system and the influence of equipment variables on image quality Proceedings of International Conference "Medical Physics in the Baltic States" vol 6913, ed J Hsieh and E Samei p 69134R

[38] Kepler K and Vladimirov A 2008 Optimisation strategies introduced for CR at health care centres in Estonia Radiat. Prot. Dosimetry 129 127-31

[39] Bath M 2010 Evaluating imaging systems: practical applications Radiat. Prot. Dosimetry $13926-36$

[40] McAdams H P, Samei E, Dobbins J, Tourassi G D and Ravin C E 2006 Recent Advances in Chest Radiography Radiology 241 663-83

[41] Håkansson M, Båth M, Börjesson S, Kheddache S, Allansdotter Johnsson Å and Månsson L G 2005 Nodule detection in digital chest radiography: Effect of system noise Radiat. Prot. Dosimetry 114 97-101

[42] Fausto A M F, Lopes M C, de Sousa M C, Furquim T A C, Mol A W and Velasco F G 2017 Optimization of Image Quality and Dose in Digital Mammography J. Digit. Imaging 30 185-96 
[43] Rojas L J, Fausto A M F, Mol A W, Velasco F G, Abreu P O S, Henriques G and Furquim T A C 2017 Optimization of the exposure parameters in digital mammography using contrast-detail metrics Phys. Medica 42 13-8 